\title{
INFANTILE ALEXANDER DISEASE WITH LATE ONSET INFANTILE SPASMS AND HYPSARRHYTHMIA
}

\author{
Paprocka ${ }^{1, *}$, Rzepka-Migut B ${ }^{2}$, Rzepka N ${ }^{2}$, Jezela-Stanek A ${ }^{3}$, Morava $E^{4}$ \\ *Corresponding Author: Dr. Justyna Paprocka, Department of Paediatric Neurology, School of Medi- \\ cine in Katowice, Medical University of Silesia, Medyków 16, 40-752 Katowice, Poland. Tel: +48-606- \\ 415-888. Fax: +48-322-071-615. E-mail: justyna.paprocka@interia.pl
}

\begin{abstract}
Alexander disease $(\mathrm{AxD})$ is a rare autosomal dominant leukodystrophy with three clinical subtypes: infantile, juvenile and adult. Forms differ by age of symptoms occurrence and the clinical presentation. Although recent data suggest considering only two subtypes: type I (infantile onset with lesions extending to the cerebral hemispheres); type II (adult onset with primary involvement of subtentorial structures). Dominant mutations in the glial fibrillary acidic protein (GFAP) gene in AxD cause dysfunction of astrocytes (a type III intermediate filament). The authors discuss the clinical picture of a boy with infantile form of AxD confirmed by the presence of de novo heterozygous mutation c. $236 \mathrm{G}>\mathrm{A}$ in the GFAP gene and without striking symptoms such as macrocephaly and with exceptional late-onset epileptic spasms with hypsarrhyth- mia on electroencephalogram (EEG).
\end{abstract}

Keywords: Alexander disease (AxD); Children; Macrocephaly.

\section{INTRODUCTION}

Alexander disease $(\mathrm{AxD})$ is a rare autosomal dominant leukodystrophy with three clinical subtypes: infantile, juvenile and adult. The neonatal forms were also described

\footnotetext{
${ }^{1}$ Department of Paediatric Neurology, School of Medicine, Medical University of Silesia, Katowice, Poland

2 Department of Paediatric Neurology, St. Queen Jadwiga's Regional Clinical Hospital, No. 2, Rzeszów, Poland

${ }^{3}$ Department of Genetics and Clinical Immunology, National Institute of Tuberculosis and Lung Diseases, Warsaw, Poland

${ }^{4}$ Hayward Genetics Center, Tulane University School of Medicine, New Orleans, LA, USA
}

[1-5]. Forms differ by age of symptoms occurrence and the clinical picture. Although recent data suggest considering only two subtypes: type I (infantile onset with lesions extending to the cerebral hemispheres); type II (adult onset with primary involvement of subtentorial structures). The infantile subgroup with early onset within 2 years of life is the most common. Dominant mutations in the glial fibrillary acidic protein $(G F A P)$ gene in $\mathrm{AxD}$ cause dysfunction of astrocytes (a type III intermediate filament).

The disease manifests with macrocephaly, retarded psychomotor development and seizures. Other common symptoms are: ataxia $[1,3,4,5]$, pyramidal signs $[3,4,5]$, including spasticity $[1,4]$. Magnetic resonance imaging (MRI) shows demyelinating changes mainly in the frontal lobes. Genetic background is a mutation in the GFAP gene with variable clinical features. The authors present a case of infantile onset $\mathrm{AxD}$ with normal head circumference.

Case Presentation. The authors present a boy with an unremarkable family history, a second child of non consanguineous parents, born vaginally at 41 weeks' gestation. The perinatal period was uneventful. He weighed $4280 \mathrm{~g}$ (50-90 percentile), his head circumference was $33.5 \mathrm{~cm}$ (3-10 percentile) and his birth length was $58 \mathrm{~cm}$ ( $>97$ percentile). The boy was rated 8 points in the Apgar scale. The parents were concerned about the child's delayed development: independent sitting at 9 month, first words at approximately 13 months, abnormal behavior, the boy rarely cried and had no interest in toys. Because of generalized hypotonia, the patient was rehabilitated since his 8 th month of life. Ultrasound examination of the head performed at 12 months showed enlargement of the lateral and third ventricle. Brain MRI (at the age of 13 months) confirmed internal hydrocephalus and directed suspicion to an aqueductal stenosis. Furthermore, it additionally revealed spots of impaired myelination. Areas of diffuse 
hyperintensity of white matter in the frontal lobe, within the left lentiform nucleus and in left-sided posterior part of the internal capsule (Figures 1, 2 and 3). Features of intracranial hypertension that might require neurosurgery intervention were not found. At 18 months, the boy was hospitalized due to a prolonged episode of left-sided seizures, with secondary generalization followed by a longer period of altered consciousness. At hospital admission, the head circumference was $49 \mathrm{~cm}$ (75-90 percentile). On neurological examination, meningeal symptoms were absent, hypotonia $(\mathrm{L}>\mathrm{R})$ with preserved tendon reflexes, were observed. Computed tomography of the brain excluded intracranial hypertension, the size of the lateral ventricles was comparable with previous MRI result (Figures 4 and 5). During the few next days of hospitalization, clusters of epileptic spasms occurred. Frequent myoclonia were also observed concomitant with temperature up to $38.4{ }^{\circ} \mathrm{C}$. The longest status epilepticus persisted for 8 hours. Because of exposure to Herpes virus, serum and cerebrospinal fluid examinations were performed, and Herpes infection was excluded. Electroencephalography revealed generalized paroxysmal activity, especially in temporal and occipital areas in the form of a symmetric hypsarrhythmia. Inadequate response to anticonvulsant treatment required many drug modifications and was coupled with developmental regression. Antiepileptic treatment (valproic acid and lamotrigine) managed to stop seizures for 10 months.

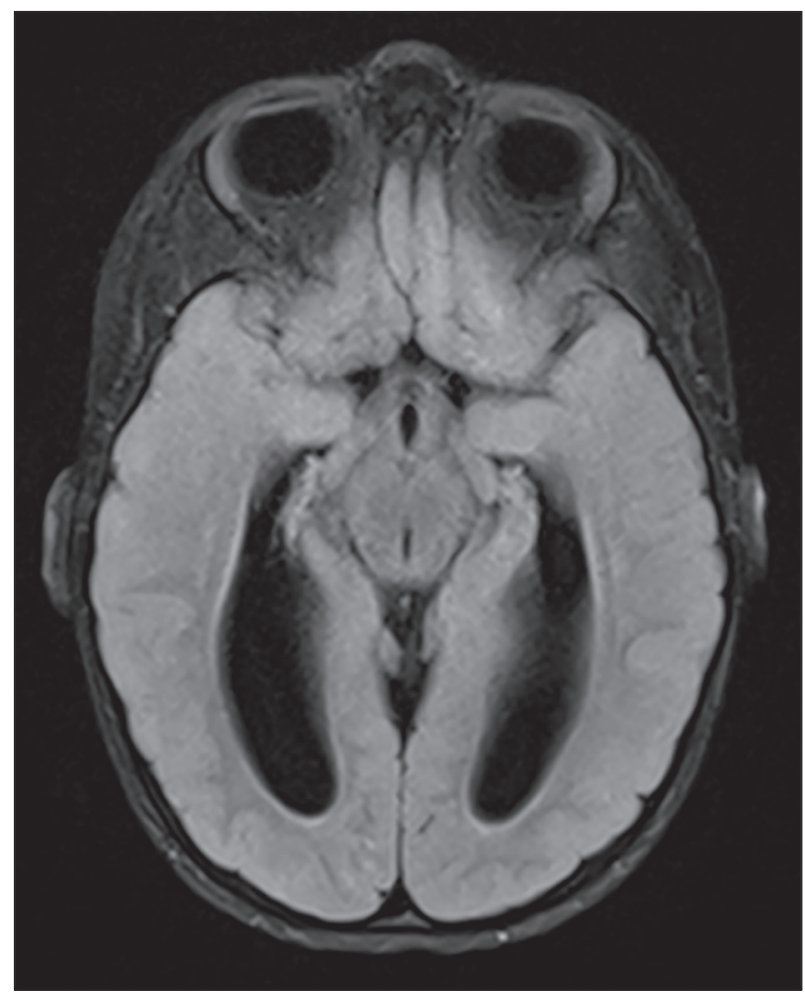

Figure 1. FLAIR transverse brain MRI (13 months) showed internal hydrocephalus and spots of impaired mye-lination.
The boy started sitting independently again, and at approximately 2 years of age, he started walking and speaking simple sentences, but the speech was unclear. Later, he was repeatedly hospitalized due to intensification of polymorphic seizures during infections. Neurological examination showed the head circumference within the range of 50-75 percentiles, hypotonia with preserved tendon reflexes, wide-based gait and ataxia. Ophthalmologic examination was normal. Based on performed metabolic work-up, organic acidurias, lysosomal storage disorders, peroxisomal disorders were excluded. Serum amino acids, acylcarnitine profile, ammonia and lactate level and thyroid function tests were within normal limits. Renal and liver function tests, hearing evaluation, ultrasonography of abdominal cavity did not show any abnormalities. Because of the presence of fever-induced seizures, mutations in the $S C N 1 A$ gene were excluded.

The neuroimaging findings were suggestive of $\mathrm{AxD}$ but macrocephaly did not occur during any period of his life. The head circumference has never exceeded 2 standard deviations. At the age of 5, molecular tests confirmed presence of a de novo heterozygous mutation (c.236G $>A$ ) in the GFAP gene, which is responsible for AxD. In the parents' samples, the described mutation was not detected. At the present time, the boy is 7 years and 8 months old and he is proceeding with an intensive rehabilitation. The neurological picture slowly deteriorates, the child presents

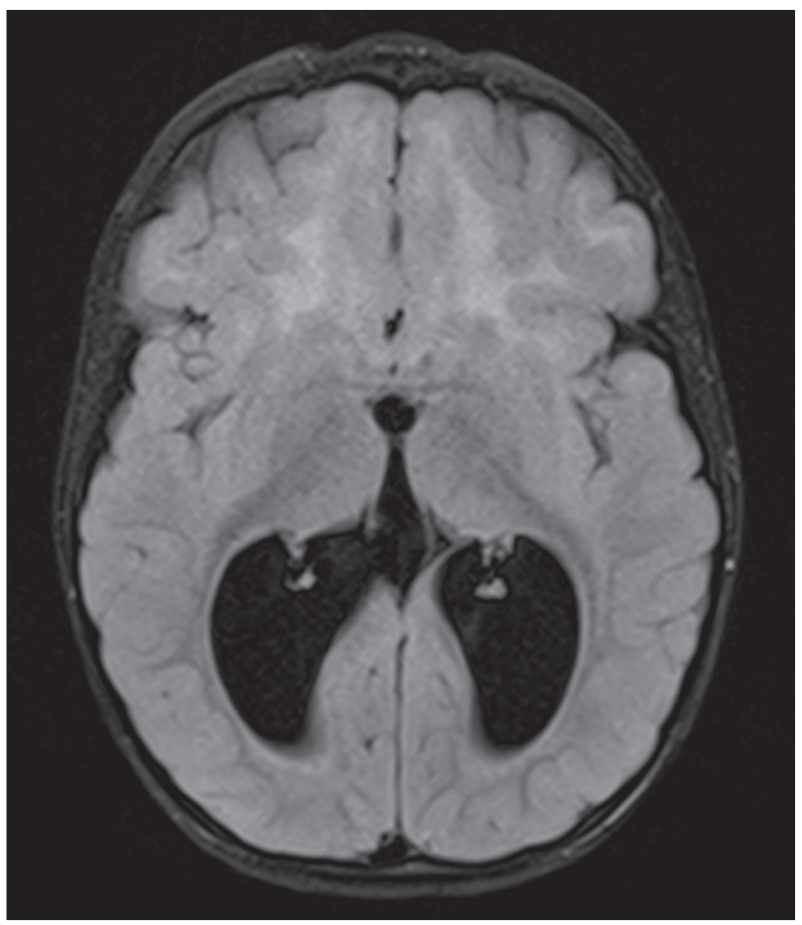

Figure 2. FLAIR transverse brain MRI (13 months) revealed ventriculomegaly with disturbed myelination: diffuse hyperintensity of white matter in the frontal lobe, within the left lentiform nucleus and in the left-sided posterior part of the internal capsule. 


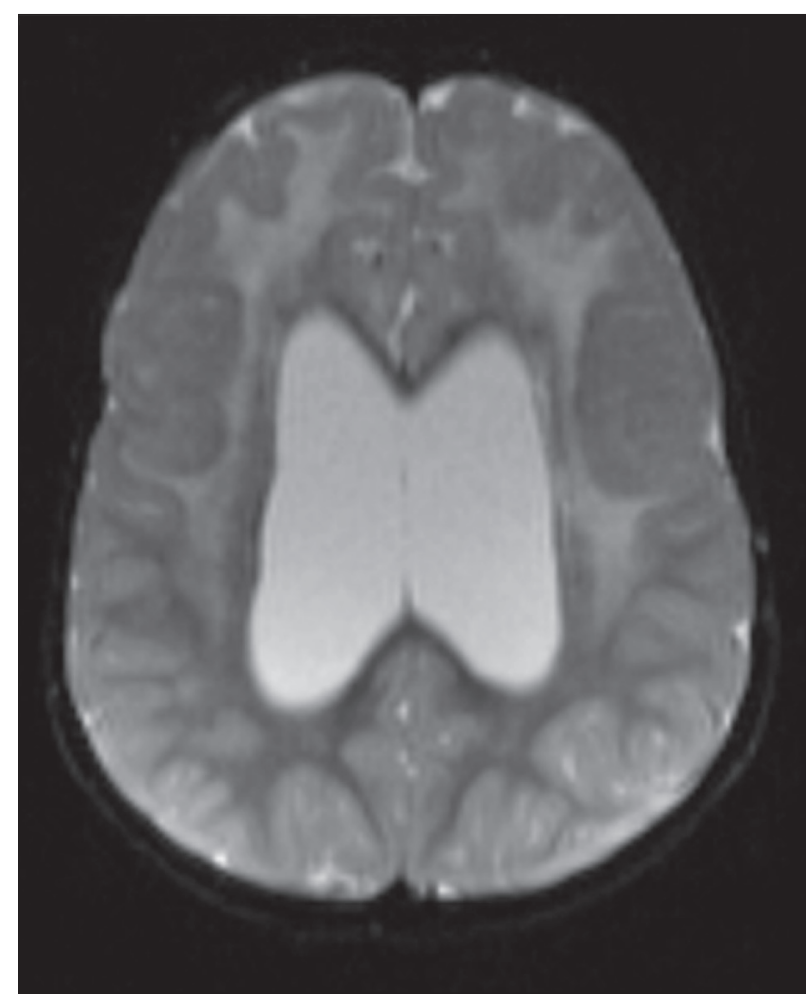

Figure 3. SET2 weighted transverse brain MRI (13 months) with areas of diffuse hyperintensity of white matter in the frontal lobe, within the left lentiform nucleus and in the left-sided part of the internal capsule.

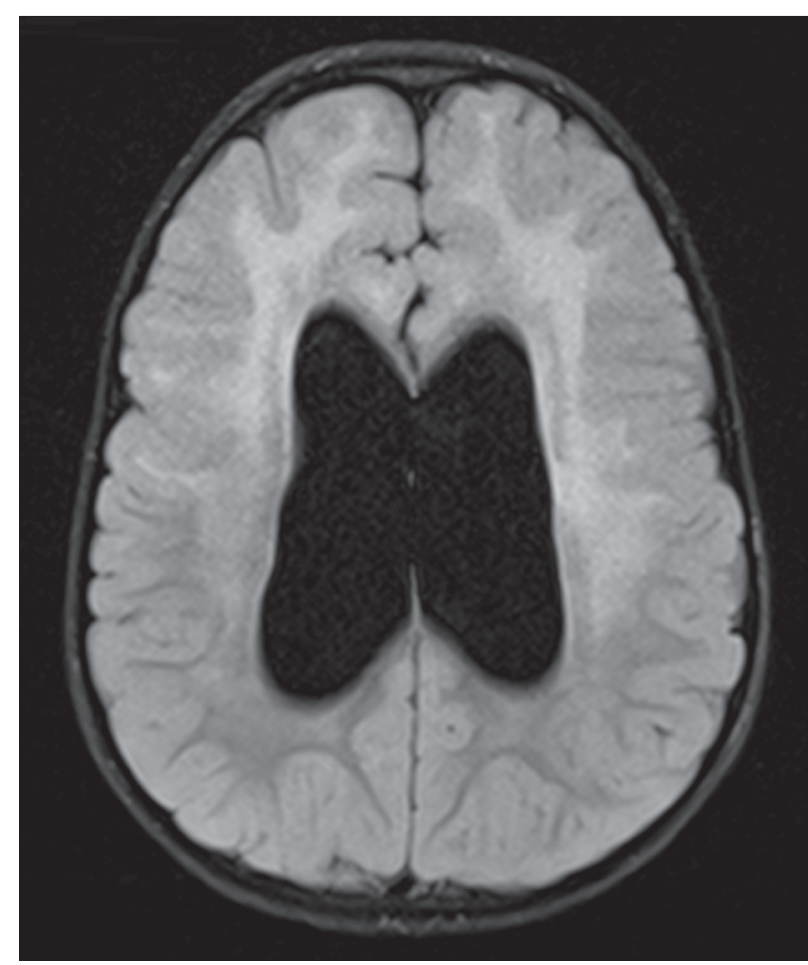

Figure 5. FLAIR transverse brain MRI (18 months) revealed internal hydrocephalus, the size of the lateral ventricles was comparable with the previous brain MRI result at the age of 13 months.

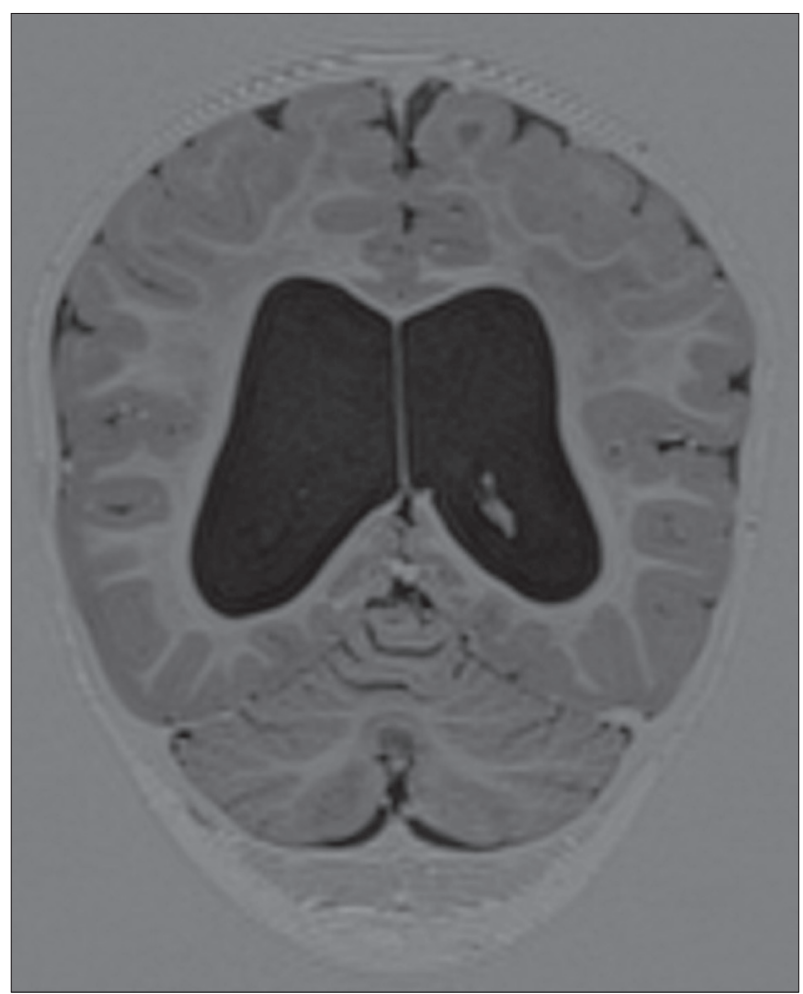

Figure 4. DICOMDIR (18 months) showed internal hydrocephalus and improper myelination compared with the previous brain MRI performed at the age of 13 months.

independent and unsteady gait, he cannot run or jump. Vocabulary is limited to simple words and speech is still unclear. He recognizes people, is friendly and open. There have been no episodes of epileptic seizures since he was 3 years old; it may be partially connected with the less number of infections, which acted as a trigger factor. Brain MRI has been repeated once a year or once in 2 years, and it is stable compared to previous examinations. The supratentorial ventricular system is dilated, not displaced and symmetrical. Hyperintensive signals are visible from white matter in the frontal lobe with fragmentary insulas and frontal parts of the external capsule in both hemispheres. Additionally, MRI showed atrophy of white matter of the occipital and parietal lobes. Focal points of incorrect signal also include head of caudate nucleus and frontal part of the left lentiform nucleus (Figure 2).

\section{DISCUSSION}

Pathomechanism of the disease is connected with a toxic gain of function mechanism, in which the mutated GFAP gene accumulates within astrocytes and aggregates with several other proteins to form inclusion bodies, known as Rosenthal fibers. Based on experimental studies there 
is a suspicion that accumulation of GFAP may lead to microglia activation and T-cell infiltration [6].

Van der Knaap et al. [7] proposed specific MRI criteria for the diagnosis of AxD including extensive, symmetric white matter abnormalities with frontal preponderance, periventricular signal changes, basal ganglia and thalamic signal changes, brainstem lesions, and contrast enhancement of multiple areas throughout the brain. The diagnosis required fulfilling at least four of five criteria. The presented boy fulfilled four of the five criteria. The most commonly used criteria were: extensive, symmetrical, cerebral white matter abnormalities with frontal preponderance, either in the extent of white matter abnormalities, the degree of swelling, the degree of signal change, or the degree of tissue loss (white matter atrophy or cystic degeneration) in 14/15 of the cases described [1-5].

One of the main goals of this study was to describe a patient with an $\mathrm{AxD}$ involving hydrocephalus without macrocephaly as well as to compare all stages of the disease with other cases in the literature. Another exceptional feature seen in our patient is late-onset epileptic spasms with hypsarrythmia. Characteristic symptoms for the infantile form are seizures, retarded psychomotor development and macrocephaly. Muscle tone abnormalities are also common symptoms. Generalized hypotonia appears in the presented case. Literature data show 15 cases of infantile form: six children had spasticity and four had hypotonia. Pyramidal symptoms, which are more common in juvenile forms, were found in the presented boy and in two other cases [2]. Hydrocephaly, which was one of the first alarming symptoms, was described only in one analyzed case [5].

Gorospe et al. [8] reported 12 genetically confirmed cases of AxD including seven with infantile onset. All had megalencephaly at presentation [1-5]. Li et al. [9] studied 26 subjects with infantile $\mathrm{AxD}$ and found macrocephaly in $62.0 \%$ of cases. In their study seizures $(92.0 \%)$ were the most common feature followed by cognitive defects $(82.0 \%)$. Bulbar signs $(62.0 \%)$, ataxia $(58.0 \%)$ and spasticity $(52.0 \%)$, were observed less often. Wilson et al. [10] described the longest survivor with $\mathrm{AxD}$, who was diagnosed with this disease at the age of 5 years and is still alive at the age of 38 .

The final AxD diagnosis was possible through results of genetic testing. Despite the fact that R79H amino acid substitution is the most common AxD variant, we did not find any case of another child with the c.236G $>$ A mutation and similar clinical outcome, improvement after treatment of seizures, in the literature [11-15]. There is also lack of reliable phenotype-genotype correlation in other GFAP gene variants. Of these, the p.Arg239His mutation was described twice $[3,4]$, and in one case macrocephaly was not found [4]. The p.Arg79His mutation was seen twice
[3] and in one case, the disease proceeded with normal head circumference [4]. The mutation p.Arg79Cys was also recorded twice [5]. Both cases were associated with hypo-tension and epileptic seizures. The R239L mutation was described twice [1,2]. Despite some description of epileptic spasms in the infancy period [16,17], the onset of this form of seizure in our patient was surprisingly late. The study was limited by the small number of publications that were compared with the gathered results. This demonstrates the importance of our work, because with more publications it would be possible to associate the type of mutation with the clinical image and the changes visible in brain MRI.

Conclusions. Alexander disease leads to severe disability and a child's death. Sometimes, with atypical presentations or course, such as in our patient. The disease is incurable and rehabilitation is the only chance to improve functioning of a child. It is essential to limit seizures and infections occurrence which may accelerate progression of the disease.

\section{ACKNOWLEDGMENTS}

We sincerely thank Professor Mario van der Knaap (Department of Child Neurology, Amsterdam, The Netherlands; Department of Functional Genomics, Center for Neurogenomics and Cognitive Research, Amsterdam Neuroscience, VU University, Amsterdam, The Netherlands) for her collaboration and assistance with data processing, MRI consultations and valuable suggestions.

Declaration of Interest. The authors report no conflicts of interest. The authors alone are responsible for the content and writing of this article.

\section{REFERENCES}

1. Tonduti D, Ardissone A, Ceccherini I, Giaccone G, Farina L, Moroni I. Unusual presentations and intrafamilial phenotypic variability in infantile onset Alexander disease. Neurol Sci. 2016; 37(6): 973-977.

2. Nishri D, Edvardson S, Lev D, Leshinsky-Silver E, Ben-Sira L, Henneke M, Lerman-Sagie T, Blumkin L. Diagnosis by whole exome sequencing of atypical infantile onset Alexander disease masquerading as a mitochondrial disorder. Eur J Paediatr Neurol. 2014; 18(4): 495-501.

3. Ashrafi MR, Tavasoli A, Aryani O, Alizadeh H, Houshmand M. Alexander disease: Report of two unrelated infantile form cases, identified by GFAP 
mutation analysis and review of literature; the first report from Iran. Iran J Pediatr. 2013; 23(4): 481-484.

4. Goyal M, Mehndiratta S, Faruq M, Dwived MK, Kapoor S. Infantile onset Alexander disease with normal head circumference: a genetically proven case report. J Clin Diagn Res. 2014; 8(11): 3-4.

5. Poloni CB, Ferey S, Haenggeli CA, Delavelle J, Bottani A, Salomons GS, et al. Alexander disease: early presence of cerebral MRI criteria. Eur J Paediatr Neurol. 2009; 13(6): 556-558.

6. Olabaria M, Putilina M, Riemer EC, Goldman JE. Astrocyte pathology in Alexander disease causes a marked inflammatory environment. Acta Neuropathol. 2015; 130(4): 469-486.

7. Van der Knaap MS, Naidu S, Breiter SN, Blaser S, Stroink H, Springer S, et al. Alexander disease: Diagnosis with MR imaging. Am J Neuroradiol. 2001; 22(3): 541-552.

8. Gorospe JR, Naidu S, Johnson AB, Puri V, Raymond GV, Jenkins SD, et al. Molecular findings in symptomatic and pre-symptomatic Alexander disease patients. Neurology. 2002; 58(10): 1494-1500.

9. Li R, Johnson AB, Salomons G, Goldman JE, Naidu $\mathrm{S}$, Quinlan R, et al. Glial fibrillary acidic protein mutations in infantile, juvenile, and adult forms of Alexander disease. Ann Neurol. 2005; 57(3): 310-326.

10. Wilson BA, Vargha-Khadem F, Florschutz G. Alexander's disease and the story of Louise. Nueropsychol Rehabil. 2018; 28(2): 199-207.

11. Brenner M, Johnson AB, Boespflug-Tanguy O, Rodriguez D, Goldman JE, Messing A. Mutations in GFAP, encoding glialfibril laryacidic protein, are associated with Alexander disease. Nat Genet. 2001; 27(1): 117120.

12. Hagemann TL1, Connor JX, Messing A. Alexander disease-associated glial fibrillary acidic protein mutations in mice induce Rosenthal fiber formation and a white matter stress response. J Neurosci. 2006; 26(43): 11162-11173.

13. Asahina N, Okamoto T, Sudo A, Kanazawa N, Tsujino S, Saitoh S. An infantile-juvenile form of Alexander disease caused by a R79H mutation in GFAP. Brain Dev. 2006; 28(2): 131-133.

14. Dotti MT, Buccoliero R, Lee A, Gorospe JR, Flint $\mathrm{D}$, Galluzzi $\mathrm{P}$, et al. An infantile case of Alexander disease unusual for its MRI features and a GFAP allele carrying both the p.Arg79His mutation and the p.Glu223Gln coding variant. J Neurol. 2009; 256(4): 679-682.

15. Lee SH, Nam TS, Kim KH, Kim JH, Yoon W, Heo $\mathrm{SH}$, et al. Aggregation-prone GFAP mutation in Alexander disease validated using a zebrafish model. BMC Neurol. 2017; 17(1): 175.

16. Torisu H, Yoshikawa Y, Yamaguchi-Takada Y, Yano T, Sanefuji M, Ishizaki Y, et al. Alexander disease with mild dorsal brain stem atrophy and infantile spasms. Brain Dev. 2013; 35(5): 441-444.

17. Lee JM, Kim AS, Lee SJ, Cho SM, Lee DS, Choi $\mathrm{SM}$, et al. A case of infantile Alexander disease accompanied by infantile spasms diagnosed by DNA analysis. J Korean Med Sci. 2006; 21(5): 954-957. 
